\title{
Reliable Low-Cost Air Quality Monitoring Using Off-The-Shelf Sensors and Statistical Calibration
}

\author{
Dejan D. Drajic*, Nenad R. Gligoric \\ DunavNET, DNET Labs, \\ Trg Oslobodjenja 127, Novi Sad, Serbia \\ dejan.drajic@dunavnet.eu
}

\begin{abstract}
Modern cities are densely populated spaces and number of people living in cities is increasing rapidly by years. The air monitoring stations exist in most of the cities to monitor air pollution. However, their number is insufficient having in mind the high cost of stations, as well as annual calibration cost. The potential solution is to use low-cost off-the-shelf sensors to monitor related air quality parameters, but they are not reliable due to the low accuracy, calibration issues, and short life cycle. In this paper, the methodology is proposed for calibration off-the-shelf air quality sensors using statistical algorithms and offset values from the official public measurement stations. The possibilities are analysed to improve the reliability of low-cost sensors by processing the obtained raw data. Special attention is devoted to the detection and elimination of short intervals when the raw results have the extraordinary high value-peaks and to the corresponding interpolation of these data. New algorithm for "peaks" detection and elimination is proposed and evaluated. Common Air Quality Index (CAQI) is calculated and evaluated in comparison with public monitoring station. It is shown that low-cost sensors could be used with high reliability as a complementary network to public monitoring stations.
\end{abstract}

Index Terms-Air pollution; CAQI calculation; Low-cost sensors; Peaks elimination.

\section{INTRODUCTION}

The population of the earth is growing up fast, but the urban population is growing up even faster. Modern cities are densely populated spaces and number of people living in cities is increasing rapidly by years. From year 2007, more than half of the world's population live in urban areas [1], and the World Health Organisation (WHO) estimates, that by year 2050 , cities will be home to more of $70 \%$ of the planet's population [2]. It is expected as well, that by 2030 , more than 27 cities will have population over 10 million [3].

In order to deal with increasing populations and enable sustained economic growth, changes to urban transport infrastructure and new housing developments are unavoidable. However, overpopulation can adversely affect the quality of life of the citizens having in mind that their

Manuscript received 19 October, 2019; accepted 8 February, 2020. This research was funded by a H2020 SynchroniCity Open Call under the name "Smart Impact". health is affected by the air pollution as one of the most significant risks for the inhabitants [4]. The WHO issued the Air Quality Guidelines [5] to direct activities in the area and to try to address the pollution challenge. Of course, there is a relevant legislation in many countries as well.

The air monitoring stations exist in most of the cities to monitor air pollution in urban areas. However, their number is insufficient having in mind the high cost per station, as well as annual calibration cost. The potential solution is to use low-cost off-the-shelf sensors to monitor related air quality parameters $(\mathrm{CO}, \mathrm{NO}, \mathrm{NO} 2, \mathrm{CO} 2, \mathrm{SO} 2, \mathrm{O} 3, \mathrm{PM})$, but they are not reliable due to the low accuracy, calibration issues, and short life cycle.

In this paper, we are proposing the methodology for calibration off-the-shelf air quality sensors using statistical algorithms and offset values from the official public measurement stations. We have analysed the possibilities to improve the reliability of low-cost sensors by processing and applying algorithms on the obtained raw data using correlation and regression methodology [6], [7]. Facilitating this theory and a regression model (interpolation and extrapolation), it has been shown that the better agreement of the processed raw-data and the results were obtained by certified (quality) instruments. The special attention is devoted to the detection and elimination of short intervals when the raw results have the extraordinary high/low valuepeaks and to the corresponding interpolation of these data. New algorithm for detection and elimination of "peaks" in measurement is proposed and evaluated, and Common Air Quality Index (CAQI) [8] is calculated and evaluated in comparison with public monitoring station.

The paper is organised as follows. In Section II, state of the art and main paper contribution are explained. In Section III, methodology of the research work and design of the algorithm are described. In section IV, the evaluation of calibration and correlation is done. Section V presents the results of evaluation for "peaks" detection algorithms and "peaks" elimination. Section VI introduces CAQI calculation and presentation of the obtained results. Section VII describes the realization of the measurement system. In Section VIII, discussions about the results and paper contribution are elaborated. Finally, Section IX provides conclusions and future ideas and work. 


\section{STATE OF THE ART AND BEYOND}

Air pollution is one of the major environmental hazard issues, and the corresponding bodies set legal pollution limits [9], [10]. Among the recognized pollutants are carbon monoxide (CO), nitrogen oxides (NO2), sulphur dioxide (SO2), ozone (O3), and particulate matter (usually PM1, PM2.5, and PM10). Temperature and air-relative humidity are monitored as well. Generally, the corresponding authorities collect the data concerning air pollution concentration. The standard procedures are used for calibration of instruments, collection of data, and the corresponding post-processing when needed.

The high quality instruments are expensive, and have a relative huge dimension and weight. For example, EN 16450 certified static PM measurement instrument [11]. Palas Fidas $200 \mathrm{~S}$ has dimensions 1810x600x400 (mm) and a weight of about $60 \mathrm{~kg}$. Further, this approach is not based on wireless networks, the measurements stations are static and sparse [4]. To obtain the tempo-spatial heterogeneity and to identify pollution hotspots require the development of the corresponding efficient real-time strategies for exposure control. Still further, regulatory decisions are formed based on long series of measurements allowing finding the temporal trends. The specific conditions related to possible hotspots must be found on available real-time data.

In the last years, low-cost sensors are becoming increasingly available to be used in air quality sensing of urban areas. In parallels, the developments in communication technologies have made the deployment of portable and relatively low-cost Micro Sensing Units (MSUs) possible. They have greater mobility, while the maintenance costs are relatively low. These sensors can be installed across the cities utilizing the existing infrastructure (e.g., attached to lampposts or installed on public transport vehicles) or even carried around by individuals contributing to crowdsource-based monitoring solutions [12]. On the other hand, they provide data with uncertain precision and should be in-field calibrated (and as well recalibrated) periodically. Moreover, wireless transmission is not always reliable and can introduce additional errors. In [4], in parallel with the thorough overview of the corresponding literature, a series of interesting questions were considered:

- Does the current state of low-cost sensing have the potential to alter the conventional way of monitoring in the future?

- Are current sensors sensitive, selective, and robust enough for reliable long-term monitoring?

- What are the associated gaps, on which future research should focus?

In [4], it is also pointed out that the gas-sensing mechanisms involve fairly complex reactions, and that the sensors performance is very sensitive to their operating conditions (e.g., temperature and relative humidity). Further, the corresponding chemical reactions in the urban atmosphere varying from daytime to night-time may additionally influence the performance of sensors. Manufacturers for temperature and relative humidity provide some correction factors. However, more sophisticated corrections are required for the outdoor conditions, where humidity and ambient temperature change significantly on diurnal, as well as on seasonal timescales.

The measurements of particulate PM using portable lowcost devices (e.g., Optical Particle Counters (OPC)) is considered in [13]. Their application under ambient conditions can be affected by high Relative Humidity $(\mathrm{RH})$ conditions. Particle-size distribution based on correction algorithm was developed to account for the influence of $\mathrm{RH}$ on sensor measurements.

The performance of sensor devices (MSUs) is usually assessed using the mean error or correlation coefficients with respect to the laboratory equipment data. In practice, on the basis of these criteria, one cannot obtain the clear insight into the performances outside of laboratory conditions or changes in performance over time. In [14], a comprehensive Sensor Evaluation Toolbox (SET) for evaluating Air Quality MSUs (AQMSUs) by a range of criteria to assess their better performance in varied applications and environments was proposed. The application of SET on measurements to the results acquired by $25 \mathrm{MSUs}$ deployed in eight cities across Europe (Project "CITY-SENSE") showed the possibilities of the proposed criteria.

The results of "CITY-SENSE" project are used as well in [15] to find the optimal calibration method of low-cost gas sensors for ambient air pollutants, where Linear Regression (LR), Multilinear Regression (MLR), and Artificial Neural Network (ANN) approaches are compared. The improvement was measured by changes in the median and interquartile ranges of statistical parameters used for model evaluation. ANN showed the best results compared to LR and MLR models. As said above, one of the biggest challenges when using low-cost sensors is the quality of measurements, which should be in accordance with Data Quality Objectives (DQO) for indicative measurements, where acceptable uncertainty is up to $25 \%$ as defined by the European legislation [10].

In [16], the focus is on London Heathrow Airport. Measurements from the sensor network were used to unequivocally distinguish airport emissions from long-range transport, and then to infer emission indices from the various London Heathrow Airport activities. The concentrations of $\mathrm{CO}, \mathrm{CO} 2$, the sum of $\mathrm{NO} 2$ and $\mathrm{O} 3$, and $\mathrm{NO}$ were measured. The results were used to create a predictive tool for modelling the corresponding pollutant concentrations. For example, the NO2 results show that the non-airport component is the dominant fraction $(\sim 75 \%)$ of annual NO2 around the airport, and that despite a predicted increase in airport related $\mathrm{NO} 2$ with an additional runway, improvements in road traffic fleet emissions are likely to more than offset this increase.

The review [17] examines the use of so-called "amperometric" electrochemical gas sensors for monitoring inorganic gases that affect urban air quality. These sensors have been well established in the industry for industrial safety since 1970s. In the review, the overview of a series of experiments is given, as well as some methods for raw data correction. During an experiment, 20 sets of CO-B4, OX$\mathrm{B} 421$, SO2-B4, NO-B4, and NO2-B4 sensors from Alphasense [18] were tested. Field-testing gave a correlation coefficient R2 of $0.9,0.73$, and 0.25 , respectively, for $\mathrm{O} 3$, 
$\mathrm{NO}$, and NO2 when compared with reference instrument measurements.

During the $1^{\text {st }}$ EuNetAir Air Quality Joint Intercomparison Exercise (Aveiro, Portugal) [19] performed from $13^{\text {th }}-27^{\text {th }}$ October 2014, the evaluation and assessment of low-cost sensors versus standard air quality reference methods were carried out. Concerning the pollutants, some promising results were observed for $\mathrm{O} 3$ (R2: 0.12-0.77), CO (R2: 0.53-0.87), and NO2 (R2: 0.02-0.89). However, for PM2.5, PM10 (R2: 0.07-0.36) and SO2 (R2: 0.09-0.20) the results showed a poor performance. In further studies [20], it was found that using post-processing and data modelling tools improved correlations were obtained between sensors and reference methods through calibration with machine learning techniques for $\mathrm{CO}(\mathrm{R} 2=0.13-0.83), \mathrm{NO} 2(\mathrm{R} 2=0.24$ $0.93)$, O3 ( R2 = 0.22-0.84), PM10 (R2 = 0.54-0.83), PM2.5 (R2 = 0.33-0.40), and SO2 (R2 = 0.49-0.84).

In [21], it was reported that after a careful calibration observed sensors met the Air Quality Directive's standards of accuracy at high concentrations of NO2. However, it was found as well that each individual sensor behaves differently meaning that each unit requires the development and application of a specific calibration model. In [22], after a series of experiments and a careful analysis of the results, a series of conclusions concerning authors' device was drawn. For example, NO/NO2/NOx comparison with ratified measurements is extremely encouraging. PM measurements capture events, but have poor scaling, especially PM10. $\mathrm{NO} 2$ is often significantly higher in absolute amounts than the reference.

Besides detailed analysis of behaviour of sensors during the long observation time ( 9 months), this paper addresses a novel algorithm for elimination of peaks in the signal for the off-the-shelf sensors for air quality monitoring. Another important contribution is the assessment of influence of air temperature and relative humidity on the measurement correlation and peak elimination. The peak elimination and calibration are then used for quantification of the CAQI air quality index based on the collected and processed measurements.

\section{METHODOLOGY}

The first step of the research was to collect the data for the analysis. Then statistical correlation is done followed by offset sensors calibration and, finally, with the signal processing, i.e. peak elimination technique. Firstly, calibration is done after 15 days of measurements, and calibration coefficients are applied on the raw measurement data. The same coefficients are used through the whole measurement campaign. Then evaluation of the peaks elimination algorithm is done to assess the efficiency of the proposed algorithm. Finally, the algorithm is deployed in a commercial platform for the measurement of air quality (ekoNET, www.solutions.net). The CAQI index is also quantified and compared between the obtained values after the calibration with index from the official monitoring stations.

The data collection was done during the period of 9 months using public air quality monitoring stations in Belgrade and a set of "low-cost" air quality sensors (Bosch BME 280, Plantower PMS 7003 (PM1, PM2.5, PM10, Alphasense gas sensors: O3-B4, NO2-B4, SO2-B4, CO-B4 [18]) deployed at the monitoring station itself. This allowed monitoring of the behavior of the sensors during the longer period, understanding influence of temperature and relative humidity on the sensor's accuracy, as well as monitoring influence of the sensors aging on measurement accuracy. Data are then statistically correlated to values captured from the official monitoring station for the exact same time intervals. In literature, there are a number of mathematical models developed for calculation of an accurate relationship between observed air pollution parameters [23]. In this paper, Least Squares Method (LSM) [6], [7] is used.

The so-called "peaks" in the measurement, represent the values of measurements in short intervals that have extraordinary high or low values in comparison to other performed measurements. The cause of this behaviour of low-cost sensors could be manifested due to the following reasons: influence of very low and high values of air temperature and relative humidity (their rapid changes) in the electronics of device (i.e., the sensitivity of electronics of a device on different kind of interferences, some micro peaks in voltage, transmission of data on local bus, interferences, etc.), the physical process of measurement work of electrochemical and optical sensor, etc. Usually peaks are singular values, but it could be sometimes a series of peaks also.

The first step would be to detect peaks and after that to eliminate the same. A special algorithm for peak detection and elimination is developed and implemented, and later applied in real-time data processing. Algorithm works as follows:

- Step 1: The basic idea is to do an averaging of measurements for the desired period and to calculate average value. The input parameter is length $L$ of the observed time period.

- Step 2: The next step is to define value $X$, which represents the lower limit for the peak value, i.e. the measurement will be candidate for a peak if its value is $X$ times higher than calculated averaged value from the step 1.

- Step 3: In order to declare that measurement is not a series of higher measurement values because of increased pollution, but a single peak, parameter $N$ is set, which represents how many consecutive values will be observed. If $N=3$, then beside "peak candidate" we observe next 3 measurements, and if only "peak candidate" has $X$ times higher value than the average value, it will be declared as a peak. Otherwise, it will be treated as a regular measurement result.

- Step 4: If the peak is detected, it will be replaced with the mean value of its previous and next measurements. In [24], it was shown how the erroneous raw data can be detected in a fully automated way, as well as that the linear interpolation showed the best performance for the gap filling for low-cost air pollution sensors. 


\section{CALIBRATION AND DATA CORRELATION}

One of the most common methods, suitable also because of the implementation simplicity, is curve fitting (calibration) using Least Squares Method (LSM) [6], [7]. Assuming that the analysed data are linearly dependent, which is fairly enough for the most of the air pollution measurements, LSM can be applied. It performs fitting based on minimization of the sum of squares of deviations from a straight-line $f(x)=a+b x$ and calculates coefficients $a$ and $b$. Let $n$ is the number of experimental points, i.e. number of conducted measurements. Denoting by $y_{i}$ the referent values (from the public monitoring station) and by $x_{i}$ the measured values (from AQ10x device, Fig. 1) the following function is formed:

$$
\begin{aligned}
L(a, b)= & \sum_{i=1}^{n}\left[y_{i}-\left(a+b x_{i}\right)\right]^{2}, \\
& \left\{\begin{array}{l}
\frac{\partial L}{\partial a}=0 \\
\frac{\partial L}{\partial b}=0 .
\end{array}\right.
\end{aligned}
$$

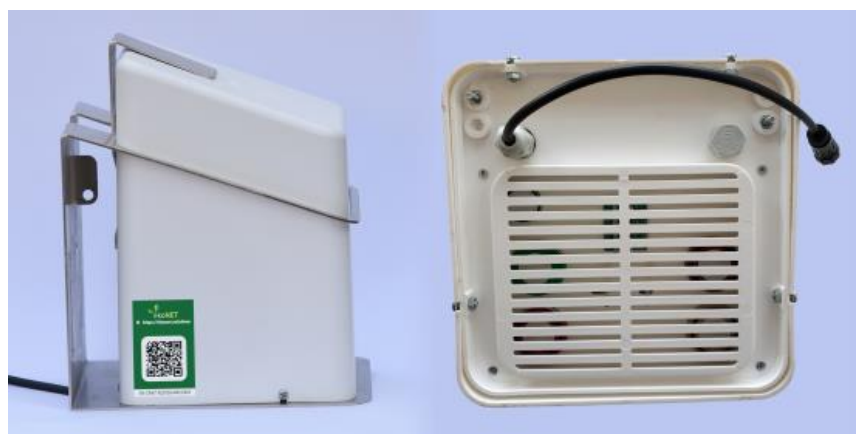

Fig. 1. Air quality ekoNET devices AQ10x (front and bottom view).

From (1) and (2) the following expressions are obtained:

$$
\begin{gathered}
n a+b \sum_{i=1}^{n} x_{i}=\sum_{i=1}^{n} y_{i}, \\
a \sum_{i=1}^{n} x_{i}+b \sum_{i=1}^{n} x_{i}^{2}=\sum_{i=1}^{n} x_{i} y_{i} .
\end{gathered}
$$

By introducing the arithmetic means:

$$
\left\{\begin{array}{l}
\bar{x}=\sum_{i=1}^{n} \frac{x_{i}}{n}, \\
\bar{y}=\sum_{i=1}^{n} \frac{y_{i}}{n},
\end{array}\right.
$$

the equations for calculation of coefficients $a$ and $b$ are obtained and "fitting" process is finished:

$$
a=\frac{\bar{y} \sum_{i=1}^{n} x_{i}^{2}-\bar{x} \sum_{i=1}^{n} x_{i} y_{i}}{\sum_{i=1}^{n} x_{i}^{2}-n \bar{x}^{2}},
$$

$$
b=\frac{\sum_{i=1}^{n} x_{i}^{2}-n \overline{x y}}{\sum_{i=1}^{n} x_{i}^{2}-n \bar{x}^{2}} .
$$

After "calibration", i.e. calculation of parameters $a$ (6) and $b$ (7), the next step is to calculate correlation of the obtained "calibrated" results with the results from public monitoring station. In order to do that, it is necessary to calculate covariance and correlation coefficient $r$.

The assessment of correlation of the collected data requires calculation of the variance:

$$
\left\{\begin{array}{l}
\sigma_{x}^{2}=\frac{1}{n} \sum_{i=1}^{n}\left(x_{i}-\bar{x}\right)^{2}, \\
\sigma_{y}^{2}=\frac{1}{n} \sum_{i=1}^{n}\left(y_{i}-\bar{y}\right)^{2},
\end{array}\right.
$$

and the covariance

$$
C(x, y)=\frac{1}{n} \sum_{i=1}^{n}\left(x_{i}-\bar{x}\right)\left(y_{i}-\bar{y}\right) .
$$

The correlation coefficient $r$ is defined as follows

$$
r=\frac{C(x, y)}{\sigma_{x} \sigma_{y}} .
$$

The correlation coefficient $r$ values range is-from -1 to +1 . The value $r=1$ implies that a linear equation describes the relationship between $x$ and $y$ perfectly. The value $r=0$ points out that there is no linear dependency. A value of -1 implies that all data points lies on a line for which $y$ decreases as $x$ increases. It is considered that the correlation is negligible if its absolute value is less than 0.3 , it is considered as medium strong when $r$ is between 0.3 and 0.7 , while it is strong when its value is over 0.7. Further, the significance of a correlation coefficient depends on the sample size. In the case when the sample size $(n)$ is relatively small (according to some authors not greater than 30 ), so called Student $(t)$ distribution is used to test significance level of correlation coefficient $r$. In our investigation, the sample size is sufficiently large, because we consider 15 days periods, and the reference values are obtained one per hour, yielding the sample size of $15 \times 24=360$.

In practice, very often results of the regression analysis are presented as the $R 2$ values

$$
R 2=R^{2}=r^{2}
$$

Calibration plots for $\mathrm{CO}$ and PM10 from referent station and from off-the-shelf sensors are provided in Fig. 2 and Fig. 3. The red line represents the "calibration line", which coefficients are calculated parameters $a$ and $b$. Also, R2 values are calculated. The compared values are averaged 
hourly values (public monitoring stations are doing measurements on every minute and these values are then averaged). It can be seen that results show quite good and high level of correlation.

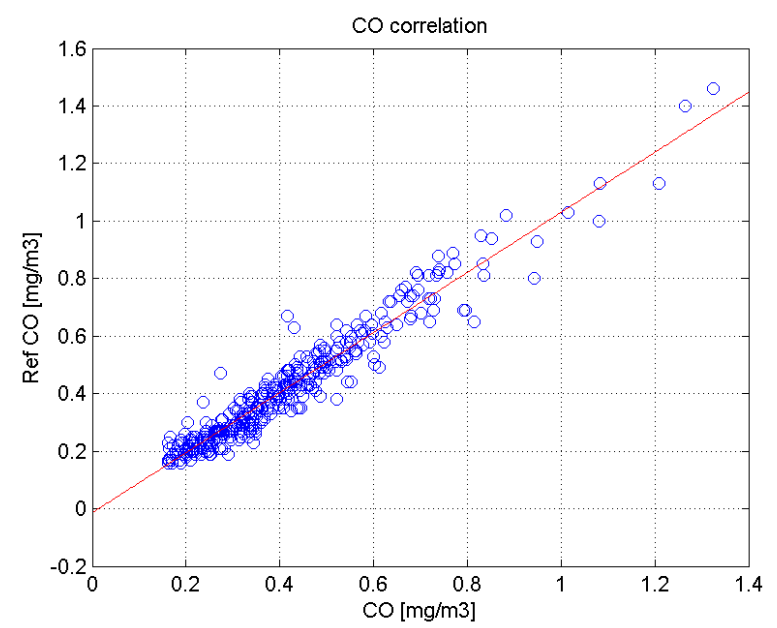

Fig. 2. CO linear correlation $(R 2=0.933)$.

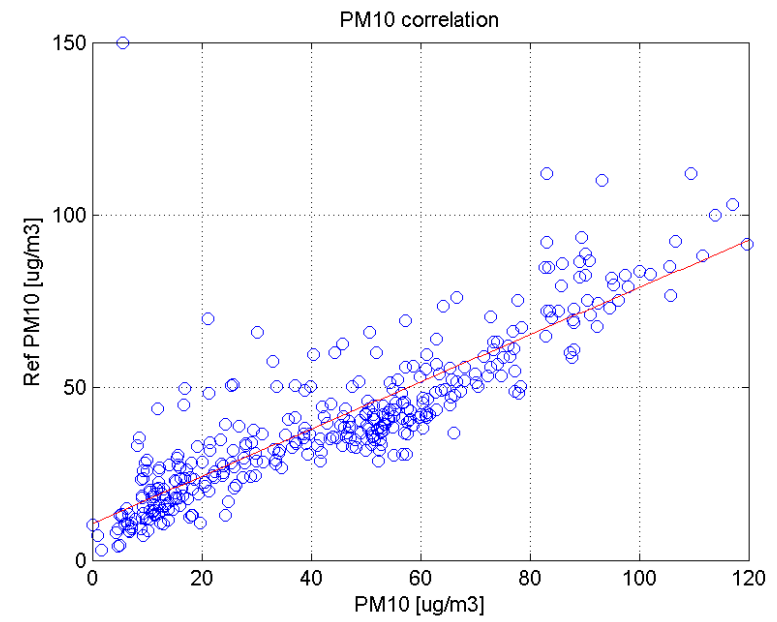

Fig. 3. PM10 linear correlation $(R 2=0.716)$.

After that, calibration coefficients are applied on the raw data and time series from public monitoring station and observed off-the-shelf sensors are compared (see Fig. 4 and Fig. 5).

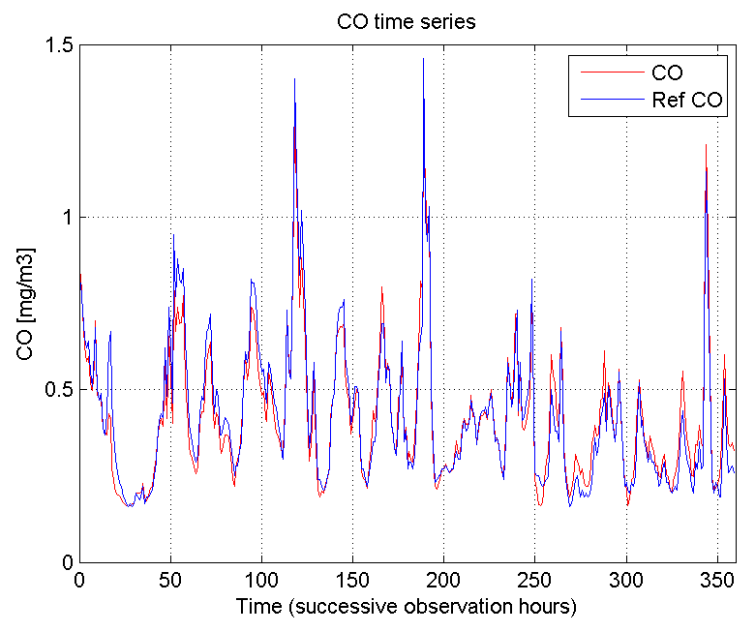

Fig. 4. Time series (360 averaged values - 15 days) comparison for $\mathrm{CO}$ (Ref CO from public monitoring station, $\mathrm{CO}$ from the observed sensor).

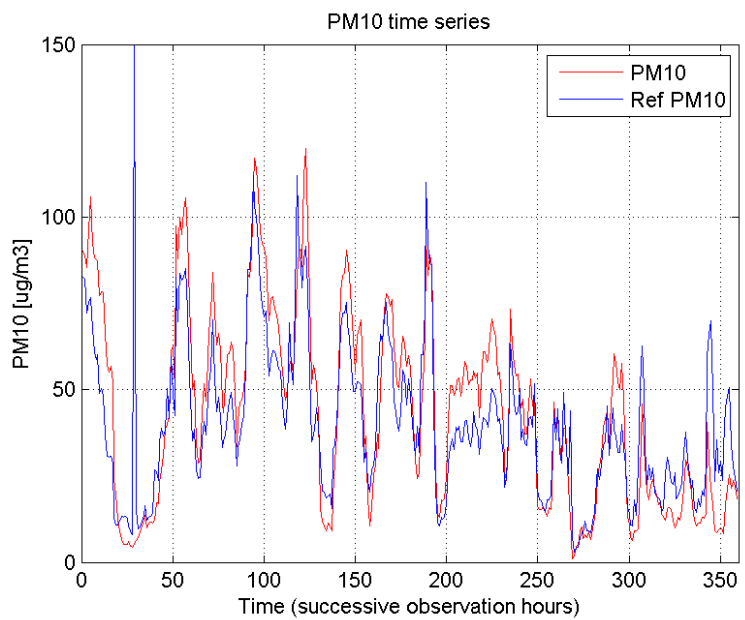

Fig. 5. Time series (360 averaged values - 15 days) comparison for PM10 (Ref PM10 from public monitoring station, PM10 from the observed sensor).

The figures show quite good overlapping of time series meaning that calibration of the sensors is done efficiently.

In Table I, R2 coefficients are given for all observed gases collected during 4 different parts of the year. The goal was to monitor behavior of the sensors through time and understand the influence of air temperature and relative humidity on the measurement correlation. As mentioned before, calibration curves defined in February remained the same during the whole observed period (October).

TABLE I. COEFICIENTS OBTAINED FOR OBSERVED PERIODS.

\begin{tabular}{|c|c|c|c|c|}
\hline & February & April & August & October \\
\hline CO [R2] & 0.933 & 0.949 & 0.861 & 0.946 \\
\hline NO2 [R2] & 0.784 & 0.846 & 0.671 & 0.828 \\
\hline PM10 [R2] & 0.716 & 0.849 & 0.664 & 0.786 \\
\hline SO2 [R2] & 0.691 & 0.712 & 0.611 & 0.711 \\
\hline O3 [R2] & 0.622 & 0.652 & 0.571 & 0.678 \\
\hline
\end{tabular}

It could be noticed that the best performance was achieved for the $\mathrm{CO}, \mathrm{NO} 2$, and PM10, while the performances of $\mathrm{SO} 2$ and $\mathrm{O} 3$ were lower, but still highly acceptable. It is obvious that temperature and relative humidity influence the behavior of low-cost sensors quite a lot, what is visible for the period of February and August when low and high temperatures influence measurements (the lowest R2 was in August when temperatures were extremely high on average and in February when the temperatures were low). Relative humidity has influence also, especially in the period when these values are high. Extreme values of temperature (low and high) and relative humidity (high values) could cause from one side "peak" in measurements and from the other side temperature (low and high) shifts sensitivity of measurements to the lower levels, which correspondingly produces results from lower accuracy (it is visible in February and August). It should be noted that for all observed periods $r$ values are higher than 0.7, which indicate strong correlation $\left(R 2=r^{2}\right.$ in Table I). More detailed analysis on the influence of temperature and relative humidity on measurement accuracy will be part of the future work, while, in the next section, more details will be devoted to "peak" detection and elimination. 


\section{Evaluation of the PEAK Elimination AlgORITHM}

The evaluation of the algorithm is done on the same set of measurements collected over the period of observed nine months.

Peaks elimination is done for the measurements with resolution on 1 minute for the period of 15 days (in all observed time periods of year), which gives total value of 21600 measurements per observed period.

For the elimination of the peaks, based on the preliminary basic investigation, we have decided to choose the following values: $L=30$ (the length of the observed time period), $X=$ 3 (the lower limit for the peak value), and $N=3$ (number of observed consecutive values). Playing with these parameters and their influence on the peak elimination will be part of the future work. Results are presented after application of algorithm on the same dataset used in the Section IV (February) in Fig. 6. The results before peaks elimination are presented in red colour, while the results after peaks elimination are presented in blue colour.

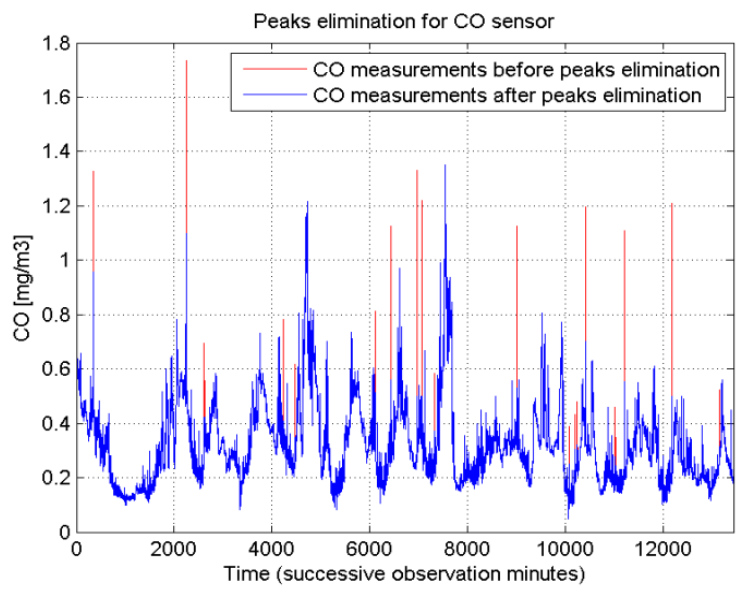

Fig. 6. Peaks elimination for CO sensor.

In Table II, statistics of the total number (No.) and percentage $(\%)$ of detected peaks for observed sensors is presented.

TABLE II. TOTAL NUMBER (No.) AND PERCENTAGE (\%) OF ELIMINATED PEAKS FOR OBSERVED PERIODS.

\begin{tabular}{|c|c|c|c|c|}
\hline & February & April & August & October \\
\hline CO [No., \%] & $\begin{array}{c}37 \\
(0.0017 \%)\end{array}$ & $\begin{array}{c}43 \\
(0.002 \%)\end{array}$ & $\begin{array}{c}57 \\
(0.0026 \%)\end{array}$ & $\begin{array}{c}38 \\
(0.0017 \%)\end{array}$ \\
\hline $\begin{array}{c}\text { NO2 [No., } \\
\%]\end{array}$ & 79 & 86 & 74 & 80 \\
$(0.0037 \%)$ & $(0.004 \%)$ & $(0.0034 \%)$ & $(0.0037 \%)$ \\
\hline $\begin{array}{c}\text { PM10 [No., } \\
\%]\end{array}$ & 33 & 19 & 79 & 11 \\
$(0.0015 \%)$ & $(0.0008 \%)$ & $(0.0037 \%)$ & $(0.0005 \%)$ \\
\hline SO2 [No., & 61 & 57 & 120 & 33 \\
$\%]$ & $(0.0028 \%)$ & $(0.0026 \%)$ & $(0.0055 \%)$ & $(0.0015 \%)$ \\
\hline O3 [No., \%] & 198 & 188 & 276 & 153 \\
$(0.0091 \%)$ & $(0.0087 \%)$ & $(0.0128 \%)$ & $(0.0071 \%)$ \\
\hline
\end{tabular}

It could be seen that sensors have different values of detected peaks, and that it depends on the observed period of year. O3 showed the highest number of detected peaks, while $\mathrm{CO}$ and PM10 had the smallest number of peaks. In the worst case, O3 has an average of one peak on every 90 minutes (observed values are generated every minute). This peak detection is of special interest for observing trends of gases and detection if some emergency measurements like a fire detection use case are considered. In the future work, it will be of interest to detect how will this peak elimination algorithm improve the correlation coefficient, which is calculated by comparing hourly averaged values, i.e. recalculate correlation coefficients calculated in Section IV with averaged values after the peak elimination.

\section{COMmon AIR QUALITY INDEX (CAQI)}

The Common Air Quality Index (CAQI) is an air quality index used in Europe since 2006 [8]. EU supported project CiteairII, evaluated CAQI on a "large set" of data. The main aim of definition and usage of CAQI was to have an index that would encourage wide comparison across EU without replacing local indices. CiteairII claimed that major motivation for creation of CAQI was an air quality index that is easy to present to the general public (visually, more understandable than ordinary numbers, since CAQI values are presented using colors that corresponds to the level of pollution, i.e. nuance of yellow, green, red) and the "main goal of the CAQI is not to warn people for possible adverse health effects of poor air quality but to attract their attention to urban air pollution and its main source (traffic) and help them decrease their exposure" [25]. Real-time frequently updated CAQI across the Europe could be found on the web site [26].

Calculated CAQI represents a number on a scale from 1 to 100 , where values $0-25$ represent very low values (means good air quality), 26-50 - low values, 51-75 - medium values, 76-100 - high values, and > 100 - very high values. CAQI could be calculated in both hourly and daily versions and separately near roads (a "roadside" or "traffic" index) or away from roads (a "background" index). Firstly, a "subindex" is calculated for each of the components $(\mathrm{CO}, \mathrm{NO} 2$, $\mathrm{SO} 2, \mathrm{O} 3$, and PM10) and final CAQI value is defined as the sub-index that represents the worst quality among those components. In Table III, a calculation scheme for CAQI is given.

TABLE III. POLLUTANTS AND CALCULATION GRID FOR THE CAQI (HOURLY AND DAILY GRID) [8].

\begin{tabular}{|c|c|c|c|c|c|c|c|c|c|c|c|c|c|}
\hline \multirow{3}{*}{$\begin{array}{l}\text { Index } \\
\text { class }\end{array}$} & \multirow{3}{*}{ Grid } & \multicolumn{6}{|c|}{ Traffic } & \multicolumn{6}{|c|}{ City Background } \\
\hline & & \multirow{2}{*}{\multicolumn{3}{|c|}{\begin{tabular}{|c|}
$\begin{array}{c}\text { Core } \\
\text { pollutants }\end{array}$ \\
NO2;PM10; \\
1h 24h
\end{tabular}}} & \multicolumn{3}{|c|}{ Pollutants } & \multicolumn{3}{|c|}{ Core pollutants } & \multicolumn{3}{|c|}{ Pollutants } \\
\hline & & & & & & & $\mathrm{CO}$ & & $\begin{array}{l}2 ; \text { PM10; } \\
1 \mathrm{~h} \mathrm{24h}\end{array}$ & 3 & & & \\
\hline Very & 0 & 0 & 0 & 0 & 0 & 0 & 0 & 0 & $\begin{array}{ll}0 & 0\end{array}$ & 0 & 0 & 0 & \\
\hline Low & 25 & 50 & 25 & 15 & 15 & 10 & 5000 & 50 & $25 \quad 15$ & 60 & 15 & 10 & 500050 \\
\hline \multirow[t]{2}{*}{ Low } & 25 & 50 & 25 & 15 & 15 & 10 & 5000 & 50 & $\begin{array}{ll}25 & 15\end{array}$ & 60 & 15 & 10 & $\begin{array}{ll}5000 \quad 50 \\
\end{array}$ \\
\hline & 50 & 100 & 50 & 30 & 30 & 20 & 7500 & 100 & $50 \quad 30$ & 120 & 30 & 20 & 7500100 \\
\hline \multirow[t]{2}{*}{ Medium } & 50 & 100 & 50 & & 30 & 20 & & 100 & $50 \quad 30$ & 120 & 30 & 20 & 10 \\
\hline & 75 & & 90 & & & 30 & 0000 & 200 & $90 \quad 50$ & 180 & 55 & 30 & 35 \\
\hline \multirow[t]{2}{*}{ High } & 75 & 200 & 90 & 50 & 55 & 30 & & 200 & $90 \quad 50$ & 180 & 55 & 30 & \\
\hline & 100 & 400 & 180 & 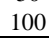 & 110 & 50 & & 400 & 180100 & 240 & 110 & 60 & 200 \\
\hline \multicolumn{2}{|c|}{$\begin{array}{l}\text { Very }>100 \\
\text { High* }^{*}\end{array}$} & \multicolumn{6}{|c|}{$>400>180>100>110>60>20000$} & \multicolumn{6}{|c|}{$>400>180>100>240>110>60>20000>500$} \\
\hline
\end{tabular}
moving average / maximum 8 hours moving average in ug/m3; PM10 - hourly value / daily value in $u \mathrm{~g} / \mathrm{m} 3$

* An index value above 100 is not calculated but reported as *>100*

More about detailed calculation of CAQI "sub-indexes" could be found in [8]. In this research, CAQI is calculated based on hourly values for the measurements taken in the city (CO, NO2, SO2, O3, PM10), which are mapped to the values $1-5$ (i.e., from very low to very high) and compared with public monitoring station measurements. Comparisons 
of CAQI calculated for these measurements and for the public monitoring station measurements are given in Table IV.

The results in Table IV should be interpreted in the following way: the CO overlaps for $100 \%$ with CAQI calculated for referent monitoring station (i.e., the same CAQI numbers 1-5), while for O3, there is between $77 \%$-$86 \%$ accuracy compared to monitoring station (O3 has more than $80 \%$ the same CAQI sub-index as referent CAQI from the monitoring station). For all compared sub-indexes, in the case that they are not the same, the difference was for only 1 index value. Sometimes our measurements had higher calculated CAQI, sometimes CAQI measurements were higher from the referent station. Total CAQI is calculated as the worst sub-index in observed hour, and sometimes the difference in sub-indexes does not affect total CAQI. For example, if the highest sub-index is 3 for both our and public monitoring station, and if other sub-indexes are different, but equal 3 or lower it will not affect total CAQI.

TABLE IV. CAQI COMPARISON FOR REFERENT MONITORING STATIONS AND OFF-THE-SHELF SENSORS.

\begin{tabular}{|c|c|c|c|c|}
\hline & February & April & August & October \\
\hline $\begin{array}{c}\text { CO sub-index } \\
\text { comparison }\end{array}$ & $100 \%$ & $100 \%$ & $100 \%$ & $100 \%$ \\
\hline $\begin{array}{c}\text { NO2 sub- } \\
\text { index } \\
\text { comparison }\end{array}$ & $88 \%$ & $97 \%$ & $84 \%$ & $95 \%$ \\
\hline $\begin{array}{c}\text { PM10 sub- } \\
\text { index } \\
\text { comparison }\end{array}$ & $83 \%$ & $85 \%$ & $81 \%$ & $83 \%$ \\
\hline $\begin{array}{c}\text { SO2 sub- } \\
\text { index } \\
\text { comparison }\end{array}$ & $96 \%$ & $97 \%$ & $92 \%$ & $97 \%$ \\
\hline $\begin{array}{c}\text { O3 sub-index } \\
\text { comparison }\end{array}$ & $84 \%$ & $86 \%$ & $77 \%$ & $85 \%$ \\
\hline CAQI & $84 \%$ & $91 \%$ & $80 \%$ & $97 \%$ \\
\hline
\end{tabular}

From the other side, it is very interesting to note, that although SO2 has a lower correlation factor R2 than NO2 and PM10, it has a better sub-index overlapping with referent station. The reason for this is because CAQI calculation is doing "quantization" of measurements in 5 levels and calculate average hourly values. The changes of $\mathrm{SO} 2$ values were not so dynamic as of NO2 and PM10 in observation analysis. CAQI calculation categorized these changes in the same range as measurements of referent stations, while for NO2 and PM10, more dynamic changes of these pollutants are measured in the observed area. Values for $\mathrm{CO}, \mathrm{O} 3$, and $\mathrm{SO} 2$ were mainly 1 and 2, while for $\mathrm{O} 3$ were in the range 1-3. Finally, for PM the range was $1-4$. In the future work, daily CAQI analysis will be calculated and analysed.

\section{REALIZATION OF THE MEASUREMENT SYSTEM}

The algorithm developed was applied in our commercial solution for air quality monitoring ekoNET (http://ekonet.solutions). The ekoNET service is designed to provide an affordable end-to-end solution for air quality monitoring leveraging Internet of Things (IoT) and cloud technologies. The service includes all necessary components: connected air quality device (AQ10x) equipped with sensors for measurement of concentration of different gases in the air, cloud based storage, and analytics engine (Microsoft Azure), as well as visualization and administration modules in the form of web and mobile applications.

The main system components are the following ones:

- Air quality monitoring devices (AQ10x device);

- Database for permanent data storage;

- Data analytics engine;

- Visualization engine;

- Administration module;

- Web and smartphone applications.

AQ10x device enables real-time monitoring of air quality and other environmental parameters. It is a portable device that can be installed both indoors and outdoors, as well as on the vehicles to enable larger coverage. Variants of AQ10x devices differ based on the communication module used (2G, 3G, LTE, NB-IoT, LoRa, SigFox, WiFi, and BLE), the set of included sensors, and the power options. The collected data is sent to the back-end server via the one of the communication modules. The data can be visualized in realtime using the appropriate web or mobile application such as shown in Fig. 7. Web and smartphone phone applications are available to visualize measurements, as well as to configure and manage the system. The current or historical data (over a selected period of time) can be visualized or exported for use in other systems. Web portal provides a rich set of tools for data processing, like filtering of results with moving average filter, averaging of results for desired period, peak elimination, etc.

AQ10x device for outdoor air quality measurements could be equipped with the following sensors: temperature, air pressure, and relative humidity sensors? Bosch BME 280, PM sensor Plantower PMS 7003 (PM1, PM2.5, PM10) or OPC-N2 Alphasense sensor, Alphasense sensors to measure concentration of gases in the air: O3-B4 (0 ppm-5 ppm), NO-B4 (0 ppm-20 ppm), NO2-B4 (0 ppm-20 ppm), SO2B4 (0 ppm-100 ppm), CO-B4 (0 ppm-50 ppm), CO2-IRCAT $(0$ ppm-5000 ppm). B4 series sensors are intended for air quality monitoring in urban, rural, and indoor areas. Monitoring of $\mathrm{CO}, \mathrm{NO} 2, \mathrm{SO} 2, \mathrm{O} 3$, and $\mathrm{PM}$ enables calculation of CAQI index. Sensors are initially calibrated during manufacturing process. However, it is necessary to perform additional calibration of all sensors. Manufacturer recommends to position the device next to a reference station with calibrated reference data (devices co-location), and to calibrate sensors based on the correlation between the measured and the reference data, what is done and presented in this paper.

\section{DISCUSSION}

The cost of the public environment monitoring equipment, which is currently used in the official measurement stations, is very high. Hence, the number of measurement points in cities around the world is rather limited. The current trend in the air quality and noise monitoring is to leverage the advancements in technology and reduction in costs to create large-scale environment monitoring deployments. 


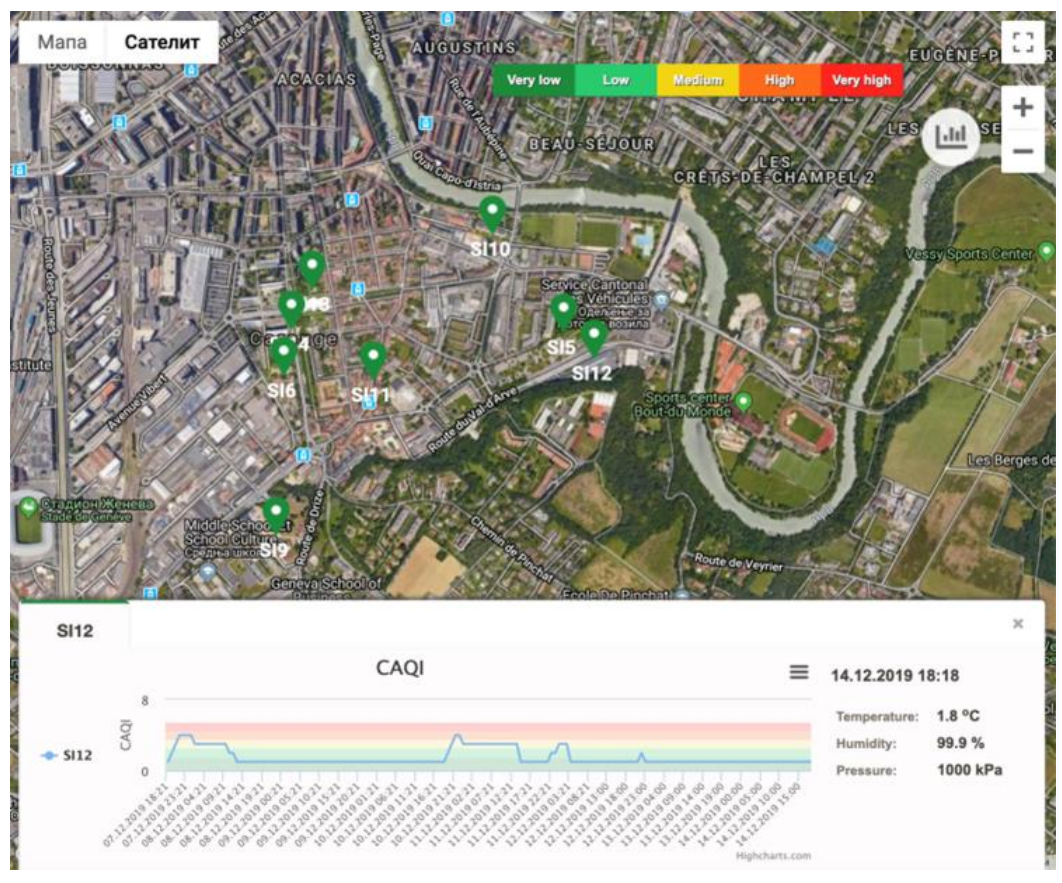

Fig. 7. Web application.

The main objective of these dense deployments is to provide a granular, indicative view of the environmental status on the scale, which was not possible before. Using this approach, interested stakeholders are able to understand the trends in air and noise pollution over a large area and then react accordingly, including deployment of mobile official measurement stations if required for legal purposes.

Modern, relatively low-cost gas sensors are used in these deployments. Their calibration is not done in labs, as such procedure would make installations prohibitively expensive. Instead, the sensors come pre-calibrated from the manufacturers and are then tuned using co-location calibration method (comparing their measurements with measurements from the official measurement stations). Examples of the correlation of the measurements obtained by the new generation of low-cost gas sensors with the measurements obtained by the official, fully calibrated, measurement station are analysed in this paper.

The measurements presented in this paper are spread over the period of 9 months (from February to October; in some occasions, there were no measurements provided from the public monitoring stations). That allowed us to make an excellent overview of the behavior of sensors in the realtime conditions, i.e. accuracy of sensors and influence of sensors aging. In general, gas-sensing mechanisms involve fairly complex reactions, and since that the performance of sensors is very sensitive to operating conditions (temperature and relative humidity). Also, the corresponding chemical reactions in the urban atmosphere varying from daytime to nighttime, may further influence the performance of sensors. Obtained correlation results are quite good. For CO: R2 = $0.861-0.933$, NO2: R2 = 0.671-0.846, PM10: R2 = 0.664$0.849, \mathrm{SO} 2: \mathrm{R} 2=0.611-0.712$, and, finally, $\mathrm{O} 2: \mathrm{R} 2=$ $0.571-0.678$. It should be noted that for all observed periods $r$ values are higher than 0.7 , which indicate strong correlation. Strong influence of temperature and relative humidity on behavior of low-cost sensors is quite visible, especially during the February and August, where corresponding low and high temperatures affects obtained measurements. Consequently, we have the lowest values of R2 in February (low temperatures in average) and August (high temperatures in average). Relative humidity has influence in the period when its values are in the higher region. As the result of these extreme values of temperature (low and high) and relative humidity (high), "peaks" in measurements could be generated. From the other side when temperatures are in these extreme regions, sensitivity of measurements is shifted to the lower levels and correspondingly obtained measurement results are with lower accuracy (that is visible in February and August measurements). Analysis of these influences will be part of the further work. Also, it would be of interest to put more devices in order to compare the behavior of different sensors separately, since they could behave slightly differently [21], and possibly cross-correlation method could be developed for calibration of higher number of collocated devices. No effects of sensors aging are noted in the observed period, but having in mind that the research was spanned for period over 9 months, we will repeat the "ageing" assessment after longer period and provide it as an updated future work.

The cause of peak occurrence for low-cost off-the-shelf sensors could be initiated by influence of very low and high values of air temperature and relative humidity in the electronics of device, the physical process of measurement work of electrochemical and optical sensor, etc. Usually peaks are singular values, but also it could be sometimes a series of peaks. These peaks could result in rising of the false alarms. Algorithm for the detection of peaks is developed, implemented, and tested. It was shown that accuracy of the sensors also depends of the observed period of the year taking into account that different period has variations in temperature and humidity. O3 showed the highest number of detected peaks, while CO and PM10 had the smallest number of peaks (CO peaks $=0.0017 \%$ $0.0026 \%, \mathrm{NO} 2$ peaks $=0.0034 \%-0.004 \%, \mathrm{PM} 10$ peaks $=$ 
$0.0005 \%-0.0037 \%, \mathrm{SO} 2$ peaks $=0.0015 \%-0.0055 \%$, and $\mathrm{O} 3$ peaks $=0.0071 \%-0.0128 \%$. Although the number of peaks was not too high, its influence should be further studied in order to see the influence of generated peaks on correlation coefficient with public monitoring station, i.e. how will the applied algorithm improve correlation coefficient. Peak elimination is of particular interest for the use cases, where device is used for observing of some phenomena, where the measurements are above predefined alarm values.

Finally, having in mind that the raw measurements are not presented to general population because people will not understand their meaning, air quality index (CAQI) is calculated using hourly and daily averages of individual measurements, thus removing reliance on individual measurements. When CAQI is calculated on hourly level and results are categorized in 5 groups (sub-indexes from 1 to 5 , which corresponds from a very low value (1 means good air quality) to very high values (5 means very bad quality)). Total CAQI represents the worst value of calculated subindexes values at the observed hour. All observed pollutants showed from good to very good correlation of CAQIs calculated for referent station and ekoNET device: $\mathrm{CO}$ $(\mathrm{CAQI}=100 \%), \mathrm{NO} 2(\mathrm{CAQI}=84 \%-97 \%)$, PM10 (CAQI $=81 \%-85 \%)$, SO2 (CAQI $=92 \%-97 \%)$, and O3 (CAQI $=77 \%-86 \%$ ). Total CAQI is in the range of $80 \%-91 \%$. Since the specific way of CAQI calculation, it is of interest to further correlate correlation coefficients and CAQI values and to perform CAQI calculation and comparisons on daily basis.

General conclusion is that measurement accuracy of every single sensor depends on the physical and chemical characteristics and has its own sensitivity to temperature, relative humidity, etc., and that for every pollutant different approaches for increasing reliability of measurements should be developed and applied. Also, it is proved that low-cost sensors could be used with high reliability as a complementary network to public monitoring stations. They can detect with very high reliability the trends that represent an indication of a potential problem. For example, if PM is continuously increasing over days, it is an indication of the problem and should result in an action undertaken by the city, such as using the official mobile measurement stations at the location of interest to get information that is more detailed. Using this approach, i.e. using cheap stations, the official air quality-monitoring network of stations is augmented, much larger granularity achieved, and the ability to observe problems at micro-locations gained.

\section{CONCLUSIONS}

In this paper, the environmental monitoring service based on the low-cost electrochemical gas sensors is presented with the algorithm for offset calibration and peak elimination using public monitoring station for obtaining referent values. Examples of the correlation of the measurements (stretched over the period of 9 months) obtained by the new generation of low-cost gas sensors with the measurements obtained by the official, fully calibrated measurement station are analysed. The proposed algorithm for "peaks" detection and elimination is developed and evaluated. The final output of the calibrated measurements forming CAQI index is also compared with the CAQI form public stations and overall results presented. The conclusion is that, with a careful data post-processing, low-cost sensors could be used with high reliability as a complementary network to public monitoring stations.

Further work will be focused on the development of calibration algorithms aiming to increase the measurement accuracy taking into account the low stability of the electrochemical gas sensors, ageing, cross-sensitivity, and impact of the air temperature and humidity on the readings. Influence of "peaks" elimination on correlation coefficient and CAQI will be studied. CAQI comparisons on 24-hour level will be calculated and evaluated.

\section{ACKNOWLEDGMENT}

We thank Ms. Daliborka Nedic for her valuable contributions in ekoNET platform development, implementation of "peaks" elimination algorithm, and support in collection of measurements and processing.

\section{CONFLICTS OF INTEREST}

The authors declare that they have no conflicts of interest.

\section{REFERENCES}

[1] State of World Population 2007. Unleashing the Potential of Urban Growth, United Nations Population Fund (UNFPA), Online report, 2007. [Online]. Available: http://www.unfpa.org/public/publications/pid/408

[2] World Health Organization (WHO), Global Health Observatory (GHO) data. [Online]. Available: https://www.who.int/gho/urban_health/situation_trends/urban_popula tion_growth_text/en/

[3] The World's Cities in 2016. Data booklet. [Online]. Available: https://www.un.org/en/development/desa/population/publications/pdf /urbanization/the_worlds_cities_in_2016_data_booklet.pdf

[4] P. Kumar et al., "The rise of low-cost sensing for managing air pollution in cities", Environment International, vol. 75, pp. 199-205, 2015. DOI: 10.1016/j.envint.2014.11.019.

[5] Air quality guidelines for Europe, 2nd ed., WHO regional publications, European series, no. 91, 2000. [Online]. Available: www.euro.who.int/document/e71922.pdf

[6] M. Engelhardt and L. J. Bain, Introduction to Probability and Mathematical Statistics. Brooks/Cole, 2000.

[7] D. Drajić, An Introduction to Statistical Communication Theory, 2nd ed. Akademska Misao, Belgrade, 2006.

[8] CiteairII: Common Information to European Air. [Online]. Available: https://www.airqualitynow.eu/download/CITEAIRComparing_Urban_Air_Quality_across_Borders.pdf

[9] Directive 98/69/EC of the European parliament and of the Council of 13 October 1998 relating to measures to be taken against air pollution by emissions from motor vehicles and amending Council Directive 70/220/EEC. O. J. Eur. Union L 350, 0001e0057.

[10] Directive 2015/1480/EC of the European Parliament and of the Council of 28 August 2015 on ambient air quality and cleaner air for Europe. O. J. Eur. Union, 2015, O. J. L 226, 29.8.2015, p. 4-11. [Online]. Available: https://eur-lex.europa.eu/legalcontent/EN/TXT/?uri=celex:32015L1480

[11] Fidas ${ }^{\circledR} 200 \mathrm{~S}$ Technical Specification. [Online]. Available: https://www.palas.de/en/product/fidas200s

[12] N. Kularatna, B. Sudantha, "An environmental air pollution monitoring system based on the IEEE 1451 standard for low cost requirements", IEEE Sensors Journal, vol. 8, no. 4, pp. 415-422, 2008. DOI: 10.1109/JSEN. 2008.917477.

[13] A. Di Antonio, O. A. M. Popoola, B. Ouyang, J. Saffell, and R. L. Jones, "Developing a relative humidity correction for low-cost sensors measuring ambient particulate matter", Sensors, vol. 18, no. 
9, p. 2790,2018 . DOI: $10.3390 / \mathrm{s} 18092790$.

[14] B. Fishbain et al., "An evaluation tool kit of air quality micro-sensing units", Science of the Total Environment, vol. 575, pp. 639-648, 2017. DOI: 10.1016/j.scitotenv.2016.09.061.

[15] D. Topalović, M. Davidović, M. Jovanović, A. Bartonova, Z. Ristovski, and M. Jovašević-Stojanović, "In search of an optimal calibration method of low-cost gas sensors for ambient air pollutants: Comparison of linear, multilinear and artificial neural network approaches", Athmosferic Environement, vol. 213, pp. 640-658, 2019. DOI: 10.1016/j.atmosenv.2019.06.028.

[16] O. A. M. Popoola et al., "Use of networks of low cost air quality sensors to quantify air quality in urban settings", Atmospheric Environment, vol. 194, pp. 58-70, 2018. DOI 10.1016/j.atmosenv.2018.09.030.

[17] R. Baron and J. Saffell, "Amperometric gas sensors as a low cost emerging technology platform for air quality monitoring applications: A review", ACS Sens., vol 2, no. 11, pp. 1553-1566, 2017. DOI: 10.1021/acssensors.7b00620.

[18] Alphasense gas sensors, Data Sheets. [Online]. Available: http://www.alphasense.com/index.php/safety/products/

[19] C. Borrego et al., "Assessment of air quality microsensors versus reference methods: The EuNetAir joint exercise", Atmospheric Environment, vol. 147, pp. 246-263, 2016. DOI: 10.1016/j.atmosenv.2016.09.050
[20] C. Borrego et al., "Assessment of air quality microsensors versus reference methods: The EuNetAir Joint Exercise - Part II", Atmospheric Environment, vol. 193, pp. 127-142, 2018. DOI 10.1016/j.atmosenv.2018.08.028.

[21] J. M. Cordero, R. Borge, and A. Narros, "Using statistical methods to carry out in field calibrations of low cost air quality sensors", Sensors and Actuators B: Chemical, vol. 267, pp. 245-254, 2018. DOI: 10.1016/j.snb.2018.04.021.

[22] D. Carruthers et al., "Using a commercial low cost sensor network (AQMesh) to quantify urban air quality: comparing measured and modelled (ADMS-urban) pollutant concentrations", Air Quality Monitoring: Evolving Issues and New Technologies, Univ. of Cambridge, 2016.

[23] P. Zanetti, Air Pollution Modeling, Theories, Computational Methods and Available Software. New York, Van Nostrand Reinhold, 1990. DOI: 10.1007/978-1-4757-4465-1.

[24] T.-B. Ottosen and P. Kumar, "Outlier detection and gap filling methodologies for low-cost air quality measurements", Environmental Science: Processes \& Impacts, vol. 21, pp. 701-713, 2019. DOI: $10.1039 /$ C8EM00593A.

[25] Air Quality Indexes. [Online]. Available: https://www.snap4city.org/drupal/node/413

[26] Air Quality Now. [Online]. Available: https://www.airqualitynow.eu/about_home.php 